\title{
Prevalence of diabetes and prediabetes (impaired fasting glucose and/or impaired glucose tolerance) in urban and rural India: Phase I results of the Indian Council of Medical Research-INdia DIABetes (ICMR-INDIAB) study
}

\author{
R. M. Anjana • R. Pradeepa • M. Deepa • M. Datta • \\ V. Sudha $\cdot$ R. Unnikrishnan $\cdot$ A. Bhansali $\cdot$ S. R. Joshi • \\ P. P. Joshi • C. S. Yajnik • V. K. Dhandhania • \\ L. M. Nath • A. K. Das • P. V. Rao • S. V. Madhu • \\ D. K. Shukla $\cdot$ T. Kaur $\cdot$ M. Priya $\cdot$ E. Nirmal . \\ S. J. Parvathi $\cdot$ S. Subhashini $\cdot$ R. Subashini $\cdot$ \\ M. K. Ali • V. Mohan • \\ on behalf of the ICMR-INDIAB Collaborative Study \\ Group
}

Received: 3 June 2011 / Accepted: 28 July 2011 /Published online: 30 September 2011

(C) Springer-Verlag 2011

\begin{abstract}
Aims/hypothesis This study reports the results of the first phase of a national study to determine the prevalence of diabetes and prediabetes (impaired fasting glucose and/or impaired glucose tolerance) in India.

Methods A total of 363 primary sampling units (188 urban, 175 rural), in three states (Tamilnadu, Maharashtra and Jharkhand) and one union territory (Chandigarh) of India were sampled using a stratified multistage sampling design to survey individuals aged $\geq 20$ years. The prevalence rates of diabetes and prediabetes were assessed by measurement of fasting and $2 \mathrm{~h}$ post glucose load capillary blood glucose. Results Of the 16,607 individuals selected for the study,
\end{abstract}

R. M. Anjana $\cdot$ R. Pradeepa $\cdot$ M. Deepa $\cdot$ M. Datta $\cdot$ V. Sudha $\cdot$

R. Unnikrishnan $\cdot$ M. Priya $\cdot$ E. Nirmal $\cdot$ S. J. Parvathi $\cdot$

S. Subhashini $\cdot$ R. Subashini $\cdot$ V. Mohan $(\square)$

Madras Diabetes Research Foundation and Dr Mohan's Diabetes

Specialities Centre, WHO Collaborating Centre for

Noncommunicable Diseases Prevention and Control,

IDF Centre for Education,

4, Conran Smith Road, Gopalapuram,

Chennai 600 086, India

e-mail: drmohans@diabetes.ind.in

\author{
A. Bhansali \\ Department of Endocrinology, Postgraduate Institute of Medical \\ Education and Research, \\ Chandigarh, India
}

14,277 (86\%) participated, of whom 13,055 gave blood samples. The weighted prevalence of diabetes (both known and newly diagnosed) was $10.4 \%$ in Tamilnadu, $8.4 \%$ in Maharashtra, 5.3\% in Jharkhand, and $13.6 \%$ in Chandigarh. The prevalences of prediabetes (impaired fasting glucose and/or impaired glucose tolerance) were $8.3 \%, 12.8 \%$, $8.1 \%$ and $14.6 \%$ respectively. Multiple logistic regression analysis showed that age, male sex, family history of diabetes, urban residence, abdominal obesity, generalised obesity, hypertension and income status were significantly associated with diabetes. Significant risk factors for prediabetes were age, family history of diabetes, abdominal obesity, hypertension and income status.

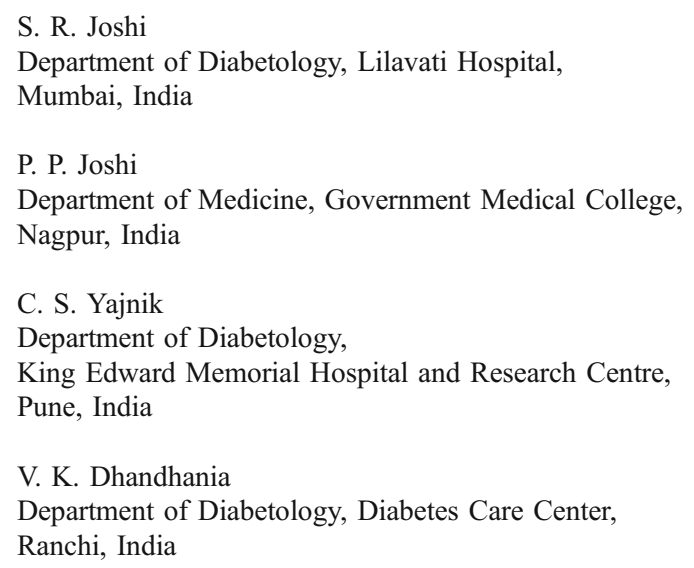


Conclusions/interpretations We estimate that, in 2011, Maharashtra will have 6 million individuals with diabetes and 9.2 million with prediabetes, Tamilnadu will have 4.8 million with diabetes and 3.9 million with prediabetes, Jharkhand will have 0.96 million with diabetes and 1.5 million with prediabetes, and Chandigarh will have 0.12 million with diabetes and 0.13 million with prediabetes. Projections for the whole of India would be 62.4 million people with diabetes and 77.2 million people with prediabetes.

Keywords Asian Indians · Diabetes · ICMR · India · INDIAB $\cdot$ Prediabetes $\cdot$ Prevalence $\cdot$ Rural $\cdot$ South Asians Urban

$\begin{array}{ll}\text { Abbreviations } & \\ \text { CBG } & \text { Capillary blood glucose } \\ \text { CEB } & \text { Census enumeration block } \\ \text { ICMR-INDIAB } & \text { Indian Council of Medical } \\ & \text { Research-INdia DIABetes } \\ \text { IFG } & \text { Impaired fasting glucose } \\ \text { IGT } & \text { Impaired glucose tolerance } \\ \text { PPS } & \text { Proportional to population size } \\ \text { PSU } & \text { Primary sampling unit } \\ \text { UT } & \text { Union territory }\end{array}$

\section{Introduction}

According to the Diabetes Atlas 2009, India has 51 million people with diabetes. However, this figure is based on a few regional studies. To date, there has been no nationwide study of diabetes in India. The Indian Council of Medical Research-INdia DIABetes (ICMRINDIAB) study was initiated, in a phased manner, to estimate the prevalence of diabetes in India. This paper presents the results of phase I of this study, involving three states and one union territory (UT), overall representing a population of 213.5 million people (18.1\% of India's population).

L. M. Nath

Association for Health Environment and Development,

New Delhi, India

\author{
A. K. Das \\ Department of Medicine, Jawaharlal Institute of Post-Graduate \\ Medical Education and Research, \\ Puducherry, India \\ P. V. Rao \\ Department of Medicine, Nizams Institute of Medical Sciences, \\ Hyderabad, India
}

\section{Methods}

The ICMR-INDIAB study methodology has been published separately [1]. This is a cross-sectional survey involving adults aged $\geq 20$ years. The results of Phase I, conducted from November 2008 to April 2010, which includes three states randomly selected to represent the south (Tamilnadu), west (Maharashtra) and east (Jharkhand) of India and one UT representing northern India (Chandigarh) are presented here.

Using a precision of $20 \%$ ( $80 \%$ power) and allowing for a non-response rate of $20 \%$, the sample size was calculated to be 4,000 per state (2,800 rural and 1,200 urban) [1], thus 16,000 for Phase I. A stratified multistage sampling design was followed [2]. The primary sampling units (PSUs) were villages in rural areas and census enumeration blocks (CEBs) in urban areas. A three-level stratification was done. A total of 16,607 individuals (5,112 urban and 11,495 rural) were selected from 363 PSUs (188 urban and 175 rural).

Institutional Ethics Committee approval and written informed consent were obtained in the local language.

\section{Data collection}

An interviewer-administered questionnaire was used and weight, height, waist and blood pressure measured using standardised techniques.

Fasting capillary blood glucose $(\mathrm{CBG})$ was determined using a One Touch Ultra glucose meter (Johnson \& Johnson, Milpitas, CA, USA). Oral glucose (82.5 g, equivalent to $75 \mathrm{~g}$ of anhydrous glucose) was given and a $2 \mathrm{~h}$ post load CBG was collected. In individuals with selfreported diabetes, only fasting $\mathrm{CBG}$ was measured.

\section{Definitions}

Diabetes was defined as individuals diagnosed by a physician and on glucose-lowering medications (selfreported) and/or those who had a fasting $\mathrm{CBG}$ $\geq 7 \mathrm{mmol} / \mathrm{l}(\geq 126 \mathrm{mg} / \mathrm{dl})$ and/or a $2 \mathrm{~h}$ post glucose $\mathrm{CBG}$ value $\geq 12.2 \mathrm{mmol} / \mathrm{l}(\geq 220 \mathrm{mg} / \mathrm{dl}) \quad$ [3]. Impaired

S. V. Madhu

Department of Medicine,

University College of Medical Sciences and GTB Hospital,

Delhi, India

D. K. Shukla $\cdot$ T. Kaur

Department of Noncommunicable Diseases,

Indian Council of Medical Research,

New Delhi, India

M. K. Ali

Madras Diabetes Research Foundation,

Chennai, India 
fasting glucose (IFG) was defined as a fasting CBG $\geq 6.1 \mathrm{mmol} / 1 \quad(\geq 110 \mathrm{mg} / \mathrm{dl})$ and $<7 \mathrm{mmol} / 1(<126 \mathrm{mg} / \mathrm{dl})$ and a $2 \mathrm{~h}$ post-glucose value $<8.9 \mathrm{mmol} / \mathrm{l}(<160 \mathrm{mg} / \mathrm{dl})$ [3]. Impaired glucose tolerance (IGT) was defined as a $2 \mathrm{~h}$ post glucose $\mathrm{CBG} \geq 8.9 \mathrm{mmol} / 1 \quad(\geq 160 \mathrm{mg} / \mathrm{dl})$ but $<12.2 \mathrm{mmol} / 1 \quad(<220 \mathrm{mg} / \mathrm{dl})$ and a fasting value $<7 \mathrm{mmol} / \mathrm{l}(<126 \mathrm{mg} / \mathrm{dl})$ [3]. Prediabetes was defined as individuals with IFG or IGT or both.

\section{Statistical analysis}

Statistical analyses were performed using SAS statistical package (version 9.0; SAS Institute, Inc., Cary, NC, USA). For all stratification, the 2001 Census of India was used. The study population was weighted for calculating prevalence rates. Weights were derived considering the design weight (reciprocal of the probability of selection) and individual response rate. The sampling weights were further normalised at the state/UT level to obtain standard state weights. The final weights were used to produce estimates of population variables. For state projections, Government of India population projections for 2011 were used [4]. For national estimates, the weighted prevalence of three states was used (the UT was excluded as it may inflate projections).

Estimates are expressed as mean \pm standard deviation or proportions. To compare continuous variables, Student's $t$ tests were used, whereas $\chi^{2}$ tests were used to test differences in proportions. Multiple logistic regression analysis was used to examine the association between various exposures and outcomes. Using backward selection, variables that remained significant were retained in the final model. A $p$ value $<0.05$ was considered significant.

\section{Results}

Of the 16,607 individuals selected for the study, 14,277 (86\%) participated, of whom 13,055 gave blood samples. There were no significant differences in demographic characteristics between the 'responders' and 'non-responders' (results not shown). The mean (SD) age was $40 \pm 14$ years, mean literacy rate $68.2 \%$ and mean monthly income 4,603 rupees (102 USD). Urban residents had significantly higher BMI, waist circumference, and systolic and diastolic blood pressures in all states after adjustment for age and sex.

Table 1 shows that the overall weighted prevalence of diabetes was $10.4 \%$ in Tamilnadu, $8.4 \%$ in Maharashtra, $5.3 \%$ in Jharkhand and $13.6 \%$ in Chandigarh. The ratio of newly diagnosed to known diabetes was more than $1: 1$ in all areas, except Tamilnadu. The overall weighted prevalences of prediabetes in Tamilnadu, Maharashtra, Jharkhand and Chandigarh were $8.3 \%, 12.8 \%, 8.1 \%$ and $14.6 \%$ respectively. This translates to 4.8 million individuals with diabetes and 3.9 million with prediabetes in Tamilnadu. In Maharashtra, an estimated 6.0 million have diabetes and 9.2 million prediabetes. Jharkhand would have 0.96 million people with diabetes and 1.5 million with prediabetes, and Chandigarh 0.12 million with diabetes and 0.13 million with prediabetes. Out of 211.6 million people who reside in the three states studied, an estimated 137 million are adults, 11.8 million of whom have diabetes and 14.6 million have prediabetes. Extrapolated to the whole country, these estimates would translate to 62.4 million individuals with diabetes and 77.2 million with prediabetes in India.

Figure 1 presents the age- and sex-specific weighted prevalence of diabetes. In all states, the take-off point in prevalence was at 25-34 years with a decline after age 65 . At every age interval, the prevalence of diabetes in urban areas was higher compared with rural areas.

Multivariable regression analyses showed that age (OR 1.7 [95\% CI 1.6,1.8, $p<0.001]$ ), male sex (OR 1.3, [95\% CI $1.1,1.5, p<0.001]$ ), family history of diabetes (OR 2.1, [95\% CI 1.7,2.6, $p<0.001]$ ), urban residence (OR 1.3, [95\% CI 1.1,1.5, $p=0.001]$ ), abdominal obesity (OR 2.4, [95\% CI 2.0,3.0, $p<0.001]$ ), generalised obesity (OR $1.6[95 \% \mathrm{CI}$ $1.3,2.0, p<0.001]$ ), hypertension (OR 1.5 [95\% CI 1.3,1.8, $p<0.001]$ ) and income status (OR 1.3 [95\% CI 1.2, 1.4, $p<0.001])$ were significantly associated with diabetes. For prediabetes, age (OR 1.2 [95\% CI 1.1,1.3, $p<0.001]$ ), family history of diabetes (OR $1.2[95 \%$ CI $1.0,1.5$, $p=0.045]$ ), abdominal obesity (OR 1.7 [95\% CI 1.4,1.9, $p<0.001]$ ), hypertension (OR $1.3[95 \%$ CI 1.1,1.5, $p=0.005]$ ) and income status (OR 1.2 [95\% CI 1.1,1.3, $p<0.001])$ were significant risk factors.

\section{Discussion}

This study is the first from India to estimate prevalence of diabetes and prediabetes, surveying rural and urban inhabitants across selected states of India. The sheer size of the populations of these states contributes to the impact of these findings. Maharashtra has a population of 112.7 million (the size of UK and Italy combined), Tamilnadu 67.4 million (the size of France) and Jharkhand 31.4 million (the size of Canada).

There have only been three multicentre studies on the prevalence of diabetes in India. The earliest study reported a prevalence of $2.1 \%$ in urban and $1.5 \%$ in rural areas [5]. The National Urban Diabetes Survey [6] showed an overall age-standardised prevalence of $12.1 \%$ for diabetes and $14 \%$ for IGT in six large metropolitan cities. The Prevalence of Diabetes in India Study [7] reported diabetes prevalence of $5.9 \%$ and $2.7 \%$ in small towns and rural areas respectively.

In our study, Chandigarh was found to have the highest prevalence of diabetes $(13.6 \%)$, which is not unexpected as 


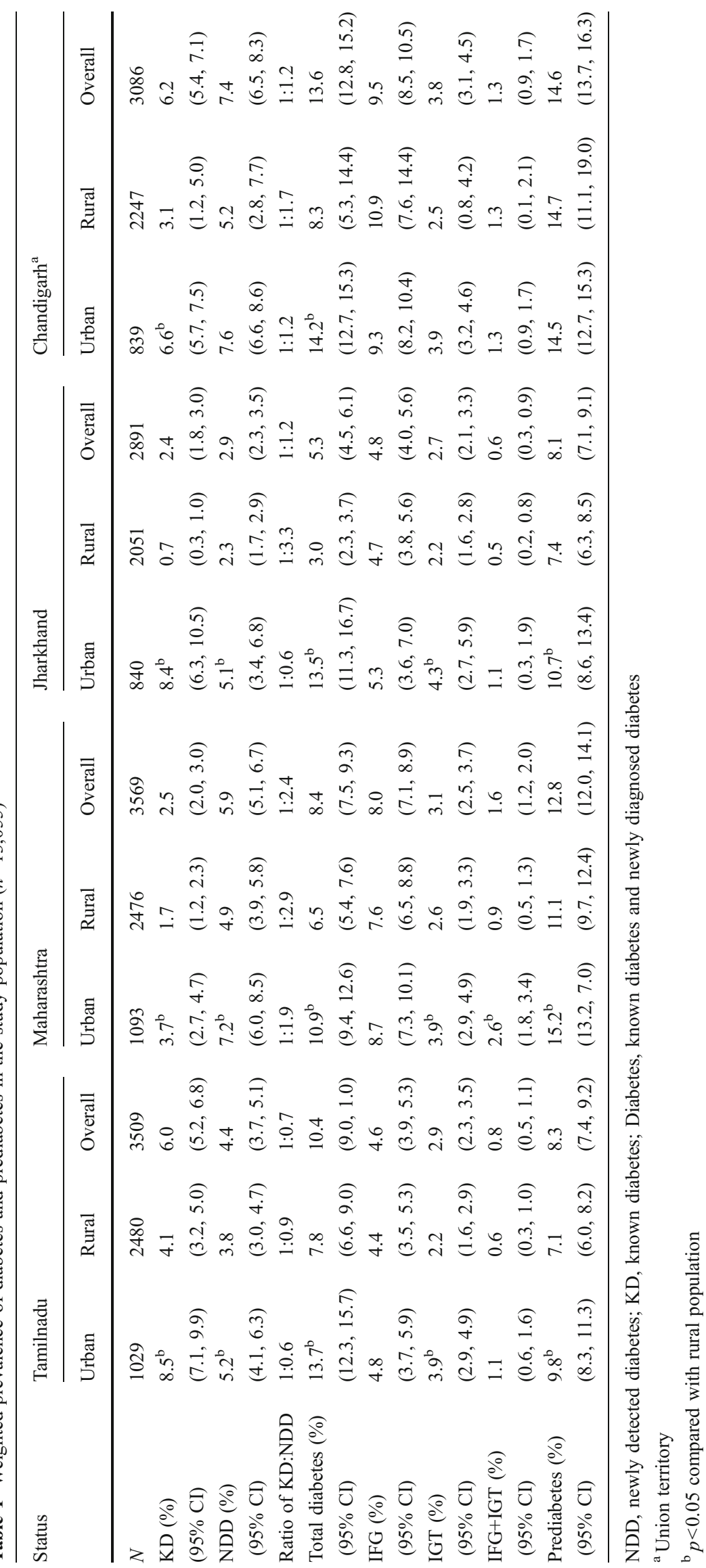


Fig. 1 Age- and sex-specific weighted prevalence of diabetes in the study population. Urban man, circles, solid line; urban woman, circles, dotted line; rural man, triangles, solid line; rural woman, triangles, dotted line. The $p$ value is the $p$ for trend across age groups in the same population; ${ }^{*} p<0.001$, $* * p<0.05$
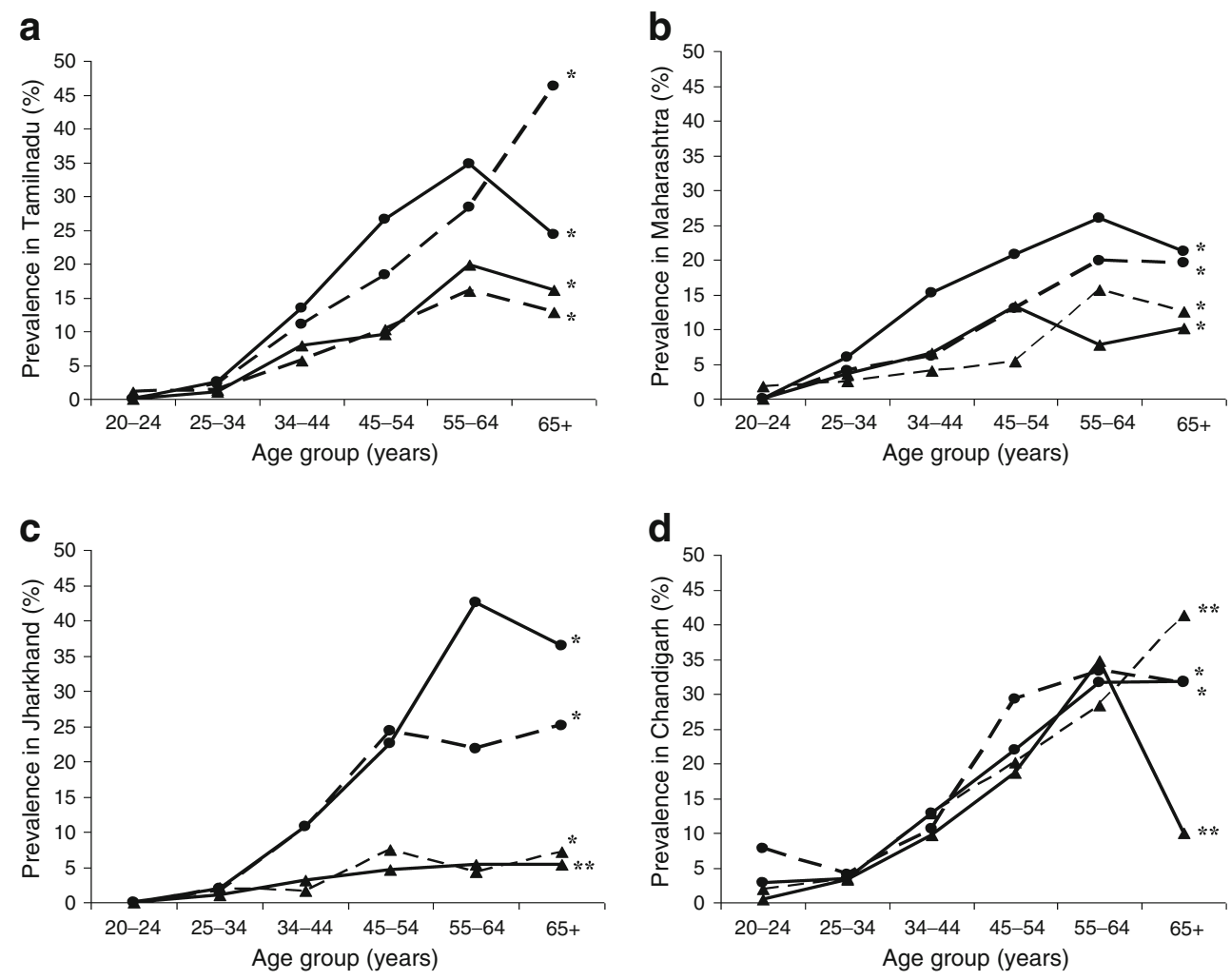

Chandigarh serves as the joint capital of Punjab and Haryana, two prosperous states in India. A previous study reported $11 \%$ prevalence in urban Chandigarh [8].

In all four states studied, the prevalence of diabetes was higher in urban, compared with rural areas. This difference was most marked in Jharkhand, where rural-urban disparities in socioeconomic status are among the highest in India. The decrease in prevalence of diabetes after 65 years is possibly due to survivor bias, possibly reflecting deaths at earlier ages due to complications of diabetes. This has significance in planning health services.

The ratio of known to newly diagnosed diabetes is a good indicator of the level of diabetes awareness in a population. In all states studied, the newly detected diabetes cases outnumbered individuals with known diabetes, except in Tamilnadu where periodic screening is done.

The prevalence of prediabetes was higher than that of diabetes in all states except Tamilnadu; the latter is probably due to quicker progression to diabetes [9] or due to ethnic differences. This calls for studies on ethnic differences between states with different risk profiles. The high prevalence of prediabetes is worrisome as this implies a huge population at risk of developing diabetes in the near future.

One of the limitations of this study is the use of CBG, which has a wider coefficient of variation than venous plasma. However, the logistical constraints of insufficient phlebotomists, poor compliance, limited availability of quality-controlled laboratories, and challenges in transporting and storing blood samples precluded the use of venous sampling. Moreover, we have shown good correlation between CBG and venous plasma estimations [10]. Also, although data from three states was used to project the number of people with diabetes in India, and final estimates may vary once the entire study is completed, the sampling frame has ensured representativeness, enhancing the credibility of our estimates.

In conclusion, this study shows that the prevalence of diabetes and prediabetes are higher in both urban and rural areas of India compared with earlier studies. With greater urbanisation, growth of the middle class and ageing of the population, we can expect huge increases in the numbers of people with diabetes in India in the future.

Acknowledgements We gratefully acknowledge the Indian Council of Medical Research, New Delhi for the financial support for the study and the ICMR-INDIAB Expert Group for their valuable suggestions and scientific inputs. We also thank the ICMR-INDIAB Quality Managers, Quality Supervisors and the field team for smooth conduct of the study and the participants for their cooperation. We thank $\mathrm{K}$. Ramachandran for his assistance in sampling and statistical inputs for the study. We thank P. Sulabha, L. Jeyaseelan and C. Ramanujam for their statistical inputs for weightage analysis. This is the second paper from the ICMR-INDIAB Study (ICMR-INDIAB-2). We acknowledge those members of the ICMR-INDIAB Core GroupMDRF (R. Jayashri, K. Velmurugan) and members of the ICMRINDIAB Expert Group (K. Ramachandran, M.D. Gupte, A. Pandey, B. Shah, R. Lakshmy) who do not appear in the author list. 
Contribution statement RMA and VM conceived the study, its design, and were involved in implementation of the study, interpretation of the data and helped to draft and revise the manuscript. RP, $\mathrm{MDe}, \mathrm{MDa}$, VS and RU were involved in the design and coordination of the study, interpretation of the data and drafting the manuscript. MKA participated in designing the study and providing critical revision of the manuscript. AB, SRJ, PPJ, CSY and VKD were responsible for supervision of the study in their respective states and were involved in the conception and design of the study. LMN, AKD, PVR, SVM, DKS and TK were part of the study expert committee and helped with the conception and design. MP, EN and SJP helped in the execution of the study, responsible for maintaining quality in the study and analysis and interpretation of data. SS and RS were responsible for data management and statistical analyses. All authors revised the manuscript critically and approved the final manuscript.

Duality of interest The authors declare that there is no duality of interest associated with this manuscript.

\section{References}

1. Anjana RM, Pradeepa R, Deepa M et al (2011) The Indian Council of Medical Research-India Diabetes (ICMR-INDIAB) Study: methodological details. J Diabetes Sci Technol 5:906-914

2. International Institute for Population Sciences (IIPS) and Macro International (2007) National Family Health Survey (NFHS-3), 2005-06: India: volume II. IIPS, Mumbai
3. World Health Organization (2006) Definition and diagnosis of diabetes mellitus and intermediate hyperglycemia: Report of a WHO/IDF Consultation, Geneva, World Health Organization, p 39

4. Population projections for India and States 2001-2026 (2006) Report of the technical group on population projections constituted by the National Commission on Population. Office of the Registrar General \& Census Commissioner, New Delhi, India, pp 140-247

5. Ahuja MMS (1979) Epidemiological studies on diabetes mellitus in India. In: Ahuja MMS (ed) Epidemiology of diabetes in developing countries. Interprint, New Delhi, pp 29-38

6. Ramachandran A, Snehalatha C, Kapur A et al (2001) High prevalence of diabetes and impaired glucose tolerance in India: National Urban Diabetes Survey. Diabetologia 44:1094-1101

7. Sadikot SM, Nigam A, Das S et al (2004) The burden of diabetes and impaired glucose tolerance in India using the WHO 1999 criteria: Prevalence of Diabetes in India Study (PODIS). Diabetes Res Clin Pract 66:301-307

8. Ravikumar P, Bhansali A, Ravikiran M et al (2010) Prevalence and risk factors of diabetes in a community-based study in North India: the Chandigarh Urban Diabetes Study (CUDS). Diabetes Metab 37:216-221

9. Mohan V, Deepa M, Deepa R et al (2006) Secular trends in the prevalence of diabetes and glucose tolerance in urban South Indiathe Chennai Urban Rural Epidemiology Study (CURES-17). Diabetologia 49:1175-1178

10. Priya M, Anjana RM, Pradeepa R et al (2011) Comparison of capillary whole blood versus venous plasma glucose estimations in screening for diabetes mellitus in epidemiological studies in developing countries. Diabetes Technol Ther 13:586-591 